preservation of myocardium by offering additional effect on modulating mPTP opening.

Methods Anesthetized open-chest rabbits underwent 1.5-h regional ischaemia/1.5-h reperfusion and were divided into four groups: control(C), preconditioning (Pre-con), gradual reperfusion (GR), and preconditioning plus gradual reperfusion (Pre-con+ GR). Control hearts underwent no additional intervention. Preconditioning consisted of three cycles of $5 \mathrm{~min}$ of ischaemia and $5 \mathrm{~min}$ of reperfusion before the 1.5-h ischaemia. Gradual reperfusion hearts underwent 5 stages involving 10 -s occlusion/50-s reperfusion, 20-s occlusion/40-s reperfusion, 30-s occlusion/30-s reperfusion, 40-s occlusion/20-s reperfusion, 50 -s occlusion/10-s reperfusion starting $10 \mathrm{~s}$ after release of the index coronary occlusion. Preconditioning plus gradual reperfusion performed both interventions in preconditioning and gradual reperfusion. $1.5 \mathrm{~h}$ reperfusion later, mitochondria were isolated from the risk region myocardium, and mPTP opening was determined by using the mPTP kinetics method.

Results Preconditioning, and gradual reperfusion alone significantly limited infarct size, which averaged $7.21 \pm 4.76 \%, 5.36 \pm 1.90 \%$ of left ventricular weigh, respectively, versus $11.94 \pm 3.75 \%$ in controls $(\mathrm{p}<0.05 \mathrm{vs}$ control). Preconditioning plus gradual reperfusion averaged $7.53 \pm 3.45 \%$ of left ventricular weigh offering no greater effect than preconditioning or gradual reperfusion alone $(\mathrm{p}>0.05)$. The $t_{1 / 2}$ of mPTP kinetics averaged $5.37 \pm 4.76 \mathrm{~min}, 5.27 \pm 4.76 \mathrm{~min}$, in preconditioning and gradual reperfusion, respectively, significantly higher than the value of $5.06 \pm 4.76 \mathrm{~min}$ in controls $(p<0.05)$. The $\mathrm{t}_{1 / 2}$ of $\mathrm{mPTP}$ kinetics averaged $6.62 \pm 4.76 \mathrm{~min}$ in preconditioning plus gradual reperfusion, however, has no more effect than preconditioning or gradual reperfusion alone $(\mathrm{p}>0.05)$.

Conclusions The combination of ischaemic preconditioning and gradual reperfusion has no greater effect on mitochondrial permeability pore but provides more powerful anti-ischaemic protection than either intervention alone.

\section{e0067 ASPIRIN ATTENUATES PULMONARY ARTERIAL HYPERTENSION IN RATS BY REDUCING PLASMA 5HYDROXYTRYPTAMINE LEVEL}

doi:10.1136/hrt.2010.208967.67

Shen Lan, Shen Jieyan, Bu Jun, Yuan Ancai. Renji Hospital Shanghai

Pulmonary arterial hypertension (PAH) is characterised by increasing pulmonary pressure, right ventricular failure, and death. The typical pathological changes include medial hypertrophy, intimal fibrosis and in situ thrombosis. 5-HT and other factors contributed to the development of pathologic lesions. Aspirin (ASA), the platelet aggregation inhibitor, inhibits 5-HT release from platelet. The aim of the current study was to determine the efficacy of aspirin in preventing or attenuating pulmonary hypertension. Sprague-Dawley (SD) rats injected with monocrotaline (MCT) at day 0 developed severe PAH at day 31. Rats were randomised to receive either vehicle or different dosages of aspirin (ASA $0.5 \mathrm{mg} / \mathrm{kg} / \mathrm{d}$, ASA $1 \mathrm{mg} / \mathrm{kg} / \mathrm{d}$, ASA $2 \mathrm{mg} / \mathrm{kg} / \mathrm{d}$, ASA $4 \mathrm{mg} / \mathrm{kg} / \mathrm{d}$ ). Aspirin suppressed $\mathrm{PAH}$ and increased survival rate compared with the placebo group ( $84 \%$ vs $60 \%, p<0.05)$. Aspirin treatment also reduced right ventricular hypertrophy and pulmonary arterioles proliferation. Plasma 5-HT measured by High Performance Liquid Chromatographic (HPLC) was decreased in aspirin treated PAH model. The degree of 5-HT reduction was associated with systolic pulmonary arterial pressure, right ventricular hypertrophy and wall thickness of pulmonary arterioles in rats. These results showed ASA treatment has effectively attenuated MCT-induced pulmonary hypertension, right ventricular hypertrophy and occlusion of pulmonary artery. The effects of ASA may be associated with reduction of 5 -HT.

\section{E0068 INVESTIGATION OF VERAPAMIL IN REVERSING ALTERATIONS OF CELLULAR ELECTROPHYSIOLOGY UNDERLYING VENTRICULAR ARRHYTHMIA IN DOGS WITH MULTIPLE ORGAN DYSFUNCTION SYNDROME}

doi:10.1136/hrt.2010.208967.68

${ }^{1}$ Zhang Ling, ${ }^{2}$ Song Jianguo, ${ }^{1}$ Hou Yuemei, ${ }^{1}$ Song Wei, ${ }^{1}$ Ma Yanhong. ${ }^{1}$ The First Affiliated Hospital of Xinjiang Medical University; ${ }^{2}$ The Center Hospital of Karamay

Objective The mechanism of Verapamil in reversing alterations of cellular electrophysiology underlying ventricular arrhythmia in dogs with multiple organ dysfunction syndrome (MODS) was not reported and their relationship to arrhythmogenesis was likely very limited.

Methods 12 dogs, of weight $8.67 \pm 0.75 \mathrm{~kg}$, were divided into two groups: control group $(n=6)$ and MODS group $(n=6)$. MODS lasting for $72 \mathrm{~h}$ was induced. Ventricular myocytes were enzymatically isolated. Early afterdepolarizations (EAD), action potential durations (APD) and L-type calcium currents were assessed before and after Verapamil perfusion.

Results Sinus arrhythmias in all MODS dogs $(100 \%$; 6 of $6, n=6)$ and premature ventricular beats in 4 MODS dogs $(66 \%$; 4 of $6, n=6)$ were recorded, while no arrhythmias were found in control animals. The prolongation of APD associated with decreased L-type $\mathrm{Ca}^{2+}$ currents and frequent provocation of $\mathrm{EAD}$ were the typical electrophysiological alterations in myocytes of MODS dogs. The AP prolongation was shortened, L-type calcium currents was decreased, EAD was suppressed by using Verapamil $(100 \mu \mathrm{mol} / \mathrm{l})$ in ventricular myocytes of MODS dogs ( $66 \%$; 4 of $6, n=6)$. EAD could be induced after elusion of Verapamil.

Conclusions The cellular electrophysiology changes within $72 \mathrm{~h}$ in the heart of MODS dogs were APD prolongation, markedly decreased L-type $\mathrm{Ca}^{2+}$ currents as well as frequently provoked EAD. Verapamil appears to be an effective agent in reversing the alternations of cellular electrophysiology in the early stage of MODS.

\section{e0069 THE INFLUENCE OF NETWORK BETWEEN CERVICAL VAGUS TRUNK AND FAT PADS ON SINUS NODE FUNCTION, ERP OF ATRIAL AND PULMONARY VEINS AND ATRIA FIBRILLATION}

doi:10.1136/hrt.2010.208967.69

${ }^{1}$ Zhang Ling, 'Zhang Xiaoqin, ${ }^{2}$ Song Jianguo, ${ }^{1}$ Song Wei, Ma Yanhong. ${ }^{1}$ The First Affiliated Hospital of Xinjiang Medical University; ${ }^{2}$ The Center Hospital of Karamay

Objective To investigate the mechanism of influence of network between cervical vagus trunk and fat pads on sinus node function, ERP of atria and pulmonary veins and inducibility and maintenance of atria fibrillation.

Methods $7 \mathrm{dogs}$, of weight 14 to $18 \mathrm{~kg}$, were placed under anaesthesia using sodium pentothal $30 \mathrm{mg} / \mathrm{kg}$, midazolam $0.4 \mathrm{mg} / \mathrm{kg}$ IV and $0.05 \mathrm{mg} / \mathrm{kg} / \mathrm{h}$. Bipolar electrode catheters were placed into the right atrial, right ventricular and bundle branch for mapping and stimulating. The hearts were exposed via right thoracotomy to expose the SAN-FP (sinus-atrial node fad pad) and AVN-FP (atria ventricular node fad pad). Bipolar electrodes and ten-polar electrodes were fixed on the left atrial appendage and the pulmonary veins. Comparison of sinus rate (SR), effective refractive period (ERP) of atrial and pulmonary vein, and both inducibility and maintenance of atrial fibrillation were performed before and after sequential ablation of SAN-FP and AVN-FP.

Results (1) The heart rate (HR) decreased significantly from $133.0 \pm 13.5 \mathrm{~ms}$ and $130.0 \pm 15.9 \mathrm{~ms}$ to $32.6 \pm 20.4 \mathrm{~ms}$ and $85.6 \pm 33.2 \mathrm{~ms}$ by stimulating right and left cervical vagus trunk, 
respectively, and $\mathrm{HR}$ decreased slightly from $125.2 \pm 21.3 \mathrm{~ms}$ to $102.5 \pm 4.94 \mathrm{~ms}$ by stimulation of SAN-FP, while HR was not affected by stimulating AVN-FP. The effect of stimulating cervical vagus trunk on reducing HR was partially expressed with SAN-FP ablation and totally eliminated by SAN-FP+AVN-FP combined ablation. (2) The ERP and increased ERP dispersion of atrial and pulmonaries were significantly by stimulating SAN-FP and abolished by ablating SAN-FP, while no big difference of ERP and ERP dispersion in atrial and pulomonaries was recorded when stimulation and ablation was exerted on AVN-FP; (3) Pacing at right atrial with $600 \mathrm{bpm}$, the AF was induced $60 \%$ and $18.4 \%$ by stimulating right and left cervical vagus trunk as well as $15.29 \%$ and $2.25 \%$ by stimulating SAN-FP and AVN-FP. However, with the stimulating at right and left cervical vagus trunk, the inducibility of AF was reduced to $16.8 \%$ and $6 \%$ when SAN-FP had ablated and even to $0 \%$ when AVN-FP had ablated.

Conclusion We concluded that sinus node function was adjusted mainly by stimulating at right cervical vagus trunk through AVNFP. The shorted ERP and increased ERP dispersion of atrial and pulmonary as well as AF were induced by stimulating cervical vagus trunk mainly coordinated with AVN-FP. Our study strongly suggested that AVN-FP is a very important coordinator in relation to parasympathetic dominant AF.

\section{e0070 ATORVASTATIN SUPPRESSES INFLAMIMATORY RESPONSE INDUCED BY OXLDL THROUGH INHIBITION OF ERK PHOSPHORYLATION, IКB? DEGRADATION AND COX-2 EXPRESSION IN MURINE MACROPHAGES}

doi:10.1136/hrt.2010.208967.70

Qin Shao, Lin Hong Shen, Liu Hua Hu, Jun Pu, Ben He. Department of Cardiology, Ren Ji Hospital, Medical School of Shanghai Jiao Tong University, Shanghai, People's Republic of China

Objective Macrophages crosstalk with oxidised low-density lipoprotein (oxLDL), play a critical role in the initiation, progression and subsequent stability of atherosclerotic plaques. Statins, inhibitors of HMG CoA (3-hydroxy-3-methylglutaryl coenzyme A) reductase, reduce the expression of inflammatory proteins in addition to their lipid-lowering action. However, the effect and the detailed antiinflammation mechanisms of statins in macrophages induced by oxLDL remain unclear. In the present study, we investigated the effect of atorvastatin on inflammatory response upon oxLDL stimulation in murine macrophages and analysed the underlying mechanisms

Methods Raw 264.7 macrophages were cultured and pre-treated with varying doses of atorvastatin in the absence or presence of $40 \mu \mathrm{g} / \mathrm{ml}$ oxLDL. The morphology of the cells was observed and the expression of inflammatory cytokines such as monocyte chemoattractant protein-1 (MCP-1) and tumour necrosis factor (TNF) was assayed by real-time PCR. The expression of Cyclooxygenases -2(COX-2) was performed by real-time PCR and Western blotting. MAPK phosphorylation and I $\mathrm{B}$ a degradation were determined by Western blotting. After pre-incubation with atorvastatin or PD98059, inhibitor of ERK1/2 MAPK, the expression of COX-2 was also detected by real-time PCR and Western blotting.

Results Our findings have shown that exposure of RAW264.7 cells to oxLDL, substantially changed the morphology of the cells and increased the mRNA expression of proinflammatory cytokines and chemokines including TNFa and MCP-1, approximately to 14-fold, 10-fold, respectively while pretreatment with atorvastatin resulted in a significant inhibition of the oxLDL-induced morphological alteration and inflammatory cytokines expression in a dosedependent fashion. Further investigation of the molecular mechanism revealed that oxLDL upregulated the transcription and protein expression of COX-2 in a time-dependent manner. Moreover, the activation of ERK pathway and I $\kappa \mathrm{Ba}$ degradation contribute to this effect.

Conclusions Taken together, the anti-inflammatory effect of atorvastatin is mediated through the inhibition of proinflammatory COX-2. Furthermore, suppression of ERK phosphorylation and IКBa degradation is involved in this regulation. Our findings provide novel evidence that statins suppress inflammatory response in murine macrophages induced by oxLDL, exert its anti-atherogenic actions via against inflammation beyond cholesterol-lowering effect.

\section{e0071 EFFECT OF FLUVASTATIN ON MYOCARDIAL INTERSTITIAL FIBROSIS AND CARDIAC FUNCTION IN DIABETIC RATS}

doi:10.1136/hrt.2010.208967.71

Dai Qiming, Liu Naifeng. Zhong Da Hospital

Objective To investigate effect of fluvastatin on myocardial interstitial fibrosis and cardiac function in diabetic rats.

Methods 24 male SD rats were randomly divided into three groups: normal control $(n=8)$, untreated and STZ-induced diabetic rats $(n=8)$ and diabetic rats treated with fluvastatin $(n=8)$. DM was induced in male SD rats with a single intraperitoneal (i.p.) injection of streptozotocin $50 \mathrm{mg} / \mathrm{kg}$ dissolved in $20 \mathrm{mM}$ citrate buffer ( $\mathrm{pH} 4.5$ ) overnight. Tail vein blood glucose was measured $72 \mathrm{~h}$ later and those with plasma glucose levels $\geq 16.7 \mathrm{mmol} / 1$ were considered to be diabetic. Control rats were injected $1 \mathrm{ml} / \mathrm{kg}$ body weight of $20 \mathrm{mM}$ citrate buffer ( $\mathrm{pH}$ 4.5) vehicle, and diabetic rats were treated with fluvastatin $(10 \mathrm{mg} / \mathrm{kg}$ administered orally, $\mathrm{n}=8)$. Fluvastatin were dissolved in sterile water, and administered every day via stomach tube. These rats were housed for 12 weeks with daily general checking. After 12 weeks intervention, miniature cardiac catheter was inserted into the left ventricle to conduct haemodynamic examination. Then, myocardium tissues were collected, collagen content was detected by picro-sirius red staining, immunohistochemistry was used to detect protein expression of fibronectin, real-time RT-PCR was used to detect the mRNA expression of CTGF and Western blotting was used to detect the protein expression of CTGF. RhoA activity in LV myocardial tissue of rats was determined by pull down assay.

Results By the end of the experiment, the left ventricular systolic pressure (LVSP) $(97 \pm 12 \mathrm{~mm} \mathrm{Hg}$ vs $131 \pm 21 \mathrm{~mm} \mathrm{Hg})$ and maximum rate of left ventricular $(\mathrm{LV})$ pressure rise and fall $(+\mathrm{dP} / \mathrm{dt}$ max and $-\mathrm{dP} / \mathrm{dt} \max )(4410 \pm 332 \mathrm{~mm} \mathrm{Hg} / \mathrm{s}$ vs $6465 \pm 442 \mathrm{~mm} \mathrm{Hg} / \mathrm{s}$ and $-4326 \pm 365 \mathrm{~mm} \mathrm{Hg} / \mathrm{s}$ vs- $6432 \pm 426 \mathrm{~mm} \mathrm{Hg} / \mathrm{s}$ ) were significantly lower and left ventricular end diastolic pressure (LVEDP) $(16.2 \pm 3.2 \mathrm{~mm} \mathrm{Hg}$ vs $4.8 \pm 1.2 \mathrm{~mm} \mathrm{Hg}$ ) were significantly higher in the diabetic group compared to the control group (all $\mathrm{p}<0.01$ ). Moreover, in LV myocardial tissue of diabetic rats the collagen content $(4.2 \% \pm 0.36 \%$ vs $6.4 \% \pm 0.33 \%, \quad p<0.01)$, fibronectin ( $3.12 \pm 0.30$ vs $0.95 \pm 0.33, p<0.01), m R N A$ and protein expression of CTGF $(0.86 \pm 0.10$ vs $1.37 \pm 0.24$ and $0.48 \pm 0.13$ vs $1.26 \pm 0.22$ $\mathrm{p}<0.01)$ and the activity of RhoA $(1.72 \pm 0.21$ vs $1.1 \pm 0.1, \mathrm{p}<0.01)$ were all significantly increased compared to the control rats. Administration of fluvastatin obviously improved the cardiac function of diabetic rats, attenuated fibronectin expression, mRNA and protein expression of CTGF and the activity of RhoA in LV myocardium of diabetic rats.

Conclusions Our data demonstrate that fluvastatin attenuates cardiac dysfunction and myocardial interstitial fibrosis of diabetic rat by inhibiting activity of RhoA to down-regulate the overexpression of CTGF, and Rho/Rho-kinase pathway may be an important target in the treatment of diabetic cardiomyopathy. 